\title{
Practical Stabilization for Uncertain Pseudo-Linear and Pseudo-Quadratic MIMO Systems
}

\author{
Laura Celentano \\ Università degli Studi di Napoli Federico II, Dipartimento di Informatica e Sistemistica, Napoli, Italy \\ Email: laura.celentano@unina.it
}

Received December 4, 2012; revised January 6, 2013; accepted January 14, 2013

\begin{abstract}
In this paper the problem of practical stabilization for a significant class of MIMO uncertain pseudo-linear and pseudoquadratic systems, with additional bounded nonlinearities and/or bounded disturbances, is considered. By using the concept of majorant system, via Lyapunov approach, new fundamental theorems, from which derive explicit formulas to design state feedback control laws, with a possible imperfect compensation of nonlinearities and disturbances, are stated. These results guarantee a specified convergence velocity of the linearized system of the majorant system and a desired steady-state output for generic uncertainties and/or generic bounded nonlinearities and/or bounded disturbances.
\end{abstract}

Keywords: Practical Stabilization; Linear and Nonlinear Uncertain Systems; Pseudo-Quadratic MIMO Uncertain System; Lyapunov Approach

\section{Introduction}

The problem of practical stability and stabilization for linear and nonlinear systems subject to disturbances and parametric uncertainties together with an efficient robust control has been in the past [1-10] one of the most research topic and nowadays remains actual and significant [11-21].

Indeed there exist many controlled or not systems linear but with uncertain parameters, uncertain pseudo-linear and with bounded coefficients, uncertain pseudoquadratic and with bounded coefficients, having a bounded additional term, for which not always there exists an equilibrium state.

Regarding this, consider:

- the mechanical systems with not viscous friction and/ or with revolute joints (e.g. robots),

- the electrical and/or electro-mechanical systems with ferromagnetic devices,

- many chemical, ecological, meteorological, biological and medical systems,

with possible disturbances and reference signals which are non standard (not polynomial or cisoidal).

For the above significant systems, it is important to design a control law such that, in a finite time interval, the state evolution, for all the initial conditions belonging to a specified compact set, is bounded and such that the evolution of the output (also the error signal) converges, with assigned minimum velocity, to chosen maximum values, that are bounded and not necessarily null.

In this paper a systematic method, in a more general framework with respect to the ones proposed in literature (see e.g. [1-5,8-10,13-18]), for the analysis and for the practical stabilization of a significant class of MIMO uncertain pseudo-linear and pseudo-quadratic systems, with additional bounded nonlinearity and/or bounded disturbances, is considered. In detail, by using the concept of majorant system, via Lyapunov approach, new fundamental theorems, from which derive explicit formulas and efficient algorithms to design state feedback control laws, with a possible imperfect compensation of nonlinearities and disturbances, are stated. These results guarantee a specified convergence velocity of the linearized system of the majorant system and a desired steady-state output for generic uncertainties and/or generic bounded nonlinearities and/or bounded disturbances (see also [19-21]). Finally two significant examples of application, well showing the utility and the efficiency of the proposed results, are reported.

\section{Problem Formulation and Preliminary Results}

Consider the following class of uncertain quadratic mul- 
tivariable systems

$$
\begin{aligned}
y^{(v)}=\tilde{F}_{1} y^{(v-1)}+\tilde{F}_{2} y^{(v-2)}+\cdots+\tilde{F}_{v} y \\
\left.+\left[\begin{array}{c}
y \\
\vdots \\
y^{(v-1)}
\end{array}\right]^{\mathrm{T}}\left[\begin{array}{ccc}
\tilde{F}_{1,11} & \cdots & \tilde{F}_{1,1 v} \\
\vdots & \ddots & \vdots \\
\tilde{F}_{1, v 1} & \cdots & \tilde{F}_{1, v v}
\end{array}\right]\left[\begin{array}{c}
y \\
\vdots \\
y^{(v-1)}
\end{array}\right]\right]+G u+\tilde{d}, \\
{\left.\left[\begin{array}{c}
y \\
\vdots \\
y^{(v-1)}
\end{array}\right]^{\mathrm{T}}\left[\begin{array}{ccc}
\tilde{F}_{m, 11} & \cdots & \tilde{F}_{m, 1 v} \\
\vdots & \ddots & \vdots \\
\tilde{F}_{m, v 1} & \cdots & \tilde{F}_{m, v v}
\end{array}\right]\left[\begin{array}{c}
y \\
\vdots \\
y^{(v-1)}
\end{array}\right]\right] }
\end{aligned}
$$

where: $y^{(i)}=\mathrm{d}^{i} y(t) / \mathrm{d} t^{i}, i=0,1, \cdots, v, y \in R^{m}$ is the output, $u \in R^{r}$ is the control input,

$\tilde{d}\left(t, y, \cdots, y^{(v-1)}, p\right) \in R^{m}$ models possible external signals and/or particular nonlinearities of the system, $p \in P$, with $P$ a compact set of $R^{\mu}$, is the vector of the uncertain parameters, $\tilde{F}_{i}\left(y, \cdots, y^{(v-1)}, p\right) \in R^{m \times m}$ and $\tilde{F}_{k, i j}\left(y, \cdots, y^{(v-1)}, p\right) \in R^{m \times m}$ are bounded matrices, continuous with respect to their arguments, $G\left(y, \cdots, y^{(v-1)}\right) \in R^{m \times r}$ is a matrix continuous with respect to its arguments and with rank $m$.

Now suppose that there exist the nominal values $\hat{F}_{i}$, $\hat{F}_{k, i j}, \hat{d}$ of $\tilde{F}_{i}, \tilde{F}_{k, i j}, \tilde{d}$ such that $F_{i}=\tilde{F}_{i}-\hat{F}_{i}$, $F_{k, i j}=\tilde{F}_{k, i j}-\hat{F}_{k, i j}$ are functions multilinear with respect to $p \in \wp$ and with respect to bounded function $g\left(y, \cdots, y^{(v-1)}\right) \in \Upsilon$, where

$$
\wp=\left\{p: p \in\left[p^{-}, p^{+}\right]\right\} \supseteq P \subset R^{\mu}
$$

and $\Upsilon=\left\{g: g \in\left[g^{-}, g^{+}\right]\right\} \subset R^{\eta}$ are hyper-rectangles, and such that $\|d=\tilde{d}-\hat{d}\| \leq \delta$, where $\delta$ is a constant.

Pose:

$$
\begin{aligned}
& n=v \cdot m, \\
& x=\left[\begin{array}{c}
y \\
y^{(1)} \\
\vdots \\
y^{(v-1)}
\end{array}\right] \in R^{n}, \hat{F}^{\prime}=\left[\begin{array}{llll}
\hat{F}_{v} & \hat{F}_{v-1} & \cdots & \hat{F}_{1}
\end{array}\right] \in R^{m \times n}, \\
& \hat{F}_{j}^{\prime \prime}=\left[\begin{array}{ccc}
\hat{F}_{j, 11} & \cdots & \hat{F}_{j, 1 v} \\
\vdots & \ddots & \vdots \\
\hat{F}_{j, v 1} & \cdots & \hat{F}_{j, v v}
\end{array}\right], F_{j}^{\prime \prime}=\left[\begin{array}{ccc}
F_{j, 11} & \cdots & F_{j, 1 v} \\
\vdots & \ddots & \vdots \\
F_{j, v 1} & \cdots & F_{j, v v}
\end{array}\right], \\
& \hat{F}_{j}^{\prime \prime}, F_{j}^{\prime \prime} \in R^{n \times n}, j=1,2, \cdots, m,
\end{aligned}
$$

and denote with $\left[\begin{array}{llll}F_{2, i 1} & F_{2, i 2} & \cdots & F_{2, i v}\end{array}\right] \in R^{m \times n}$, $i=1,2, \cdots, n$, the matrices whose $m$ rows are respectively the $i$-th rows of the $m$ matrices $F_{j}^{\prime \prime}$.

Then, by controlling the system (1.1) with the following state feedback control law with a partial compensa- tion

$$
\begin{aligned}
& u=-G^{\dagger}\left(\left[\begin{array}{llll}
K_{v} & K_{v-1} & \cdots & K_{1}
\end{array}\right] x+\hat{F}^{\prime} x+\left[\begin{array}{c}
x^{\mathrm{T}} \hat{F}_{1}^{\prime \prime} \\
\vdots \\
x^{\mathrm{T}} \hat{F}_{m}^{\prime \prime}
\end{array}\right] x+\hat{d}\right), \\
& G^{\dagger}=G^{\mathrm{T}}\left(G G^{\mathrm{T}}\right)^{-1},
\end{aligned}
$$

it is easy to verify that the closed-loop system turns out to be

$$
\begin{aligned}
& \dot{x}=\left[\begin{array}{ccccc}
0 & I & 0 & \cdots & 0 \\
0 & 0 & I & \cdots & 0 \\
\vdots & \vdots & \vdots & \ddots & \vdots \\
0 & 0 & 0 & \cdots & I \\
F_{v}-K_{v} & F_{v-1}-K_{v-1} & F_{v-2}-K_{v-2} & \cdots & F_{1}-K_{1}
\end{array}\right] x \\
& +\sum_{i=1}^{n}\left(x_{i}\left[\begin{array}{ccccc}
0 & 0 & 0 & \cdots & 0 \\
0 & 0 & 0 & \cdots & 0 \\
\vdots & \vdots & \vdots & \ddots & \vdots \\
0 & 0 & 0 & \cdots & 0 \\
F_{2, i 1} & F_{2, i 2} & F_{2, i 3} & \cdots & F_{2, i v}
\end{array}\right]\right) x+\left[\begin{array}{c}
0 \\
0 \\
\vdots \\
0 \\
I
\end{array}\right] d \\
& =A_{1} x+\sum_{i=1}^{n}\left(x_{i} A_{2, i}\right) x+B d \\
& y=\left[\begin{array}{llll}
I & 0 & \cdots & 0
\end{array}\right] x=C x .
\end{aligned}
$$

To develop a practical stabilization method for the system (1.4), in a more systematic and general framework, which allows to calculate in a simple manner a control law that guarantees a specified convergence velocity, the following notations, definitions and preliminary results are provided.

$$
\begin{aligned}
& \|x\|_{P}=\sqrt{x^{\mathrm{T}} P x},\|x\|=\|x\|_{I}=\sqrt{x^{\mathrm{T}} x}, \\
& S_{P, r}=\left\{x:\|x\|_{P} \leq r\right\}, C_{P, r}=\left\{x:\|x\|_{P}=r\right\}, \\
& \hat{C}_{P, r} \supseteq C_{P, r},
\end{aligned}
$$

where $P \in R^{n \times n}$ is a symmetric and positive definite (p.d.) matrix and $\hat{C}_{P, r}$ is a compact set.

Definition 1. Give the system (1.4) and a p.d. symmetric matrix $P \in R^{n \times n}$. A first-order positive system $\dot{\rho}=f(\rho, \delta), v=\eta(\rho)$, where $\rho(t)=\|x(t)\|_{P}$ and $\delta \geq\|d\|$, such that $v(t) \geq\|y(t)\|$ is said to be majorant system of the system (1.4).

Theorem 1. Consider the quadratic system

$$
\begin{aligned}
& \dot{\rho}=\alpha_{1} \rho+\alpha_{2} \rho^{2}+\beta \delta=\alpha_{2} \rho^{2}+\alpha_{1} \rho+\alpha_{0}, \\
& \alpha_{1}<0, \alpha_{2}, \beta \geq 0, \rho(0)=\rho_{0} \geq 0, \delta \geq 0 .
\end{aligned}
$$

If $\delta<\alpha_{1}^{2} / 4 \alpha_{2} \beta$, it is (see Figure 1) 


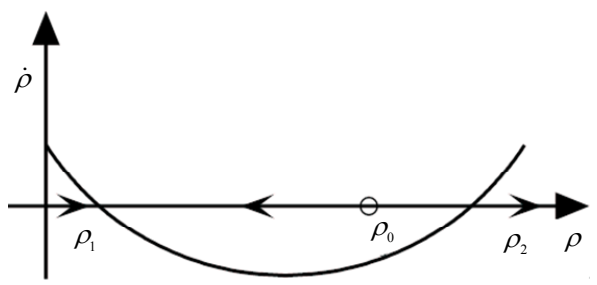

Figure 1. Graphical representation of the system (1.6).

$$
\lim _{t \rightarrow \infty} \rho(t) \leq \rho_{1}, \forall \rho_{0}<\rho_{2},
$$

where $\rho_{1}, \rho_{2}, \rho_{1}<\rho_{2}$, are the roots of the algebraic equation $\alpha_{2} \rho^{2}+\alpha_{1} \rho+\alpha_{0}=0$. Moreover for $\delta=0$ the practical convergence time $t_{5 \%} \square \rho\left(t_{5 \%}\right)=5 \% \rho_{0} \quad$ is given by (see Figure 2)

$$
\begin{aligned}
& t_{5 \%}=\gamma \tau_{l}, \tau_{l}=-1 / \alpha_{1}, \gamma=\ln \frac{20-\rho_{0} / \rho_{20}}{1-\rho_{0} / \rho_{20}}, \\
& \rho_{20}=-\alpha_{1} / \alpha_{2},
\end{aligned}
$$

in which $\tau_{l}$ is the time constant of the linearized of the system (1.6) and $\rho_{20}$ is the upper bound of the convergence interval of $\rho(t)$ for $\delta=0$, i.e. of the system (1.6) in free evolution.

Proof. The proof of (1.7) easily follows from Figure 1. Instead (1.8) easily derives by noting that the solution of (1.6) for $d=0$ is

$$
\frac{\rho(t)}{\rho_{0}}=\frac{\rho_{20}}{\rho_{0}} \frac{1}{1+\left(\frac{\rho_{20}}{\rho_{0}}-1\right) \mathrm{e}^{t / \tau_{l}}} .
$$

In Figure 2 the evolution of $\gamma$ as a function of $\rho_{20} / \rho_{0}$ is reported. By analyzing Figure 2, note that for $\rho_{20} / \rho_{0} \leq 0.27$ it is $\gamma \in[3,3.3]$, i.e. $t_{5 \%} \cong 3 \tau_{l}$.

Theorem 2. The solution of the Equation (1.6) with $\alpha_{1}^{2}-4 \alpha_{0} \alpha_{2}>0$ is

$$
\rho=\frac{\rho_{1}-\rho_{2} f}{1-f}, f=\frac{\rho_{0}-\rho_{1}}{\rho_{0}-\rho_{2}} \mathrm{e}^{\frac{\rho_{1}-\rho_{2}}{a_{2}} t},
$$

where $\rho_{1}, \rho_{2}, \rho_{1}<\rho_{2}$, are the roots of the equation $a_{2} \rho^{2}+a_{1} \rho+a_{0}=0$.

Proof. From (1.6) it derives

$$
\begin{aligned}
\frac{\mathrm{d} \rho}{\rho^{2}+\frac{a_{1}}{a_{2}} \rho+\frac{a_{0}}{a_{2}}} & =\frac{1}{\rho_{1}-\rho_{2}}\left(\frac{1}{\rho-\rho_{1}}-\frac{1}{\rho-\rho_{2}}\right) \mathrm{d} \rho \\
& =a_{2} \mathrm{~d} t,
\end{aligned}
$$

from which (1.10) easily follows.

Lemma 1. If $P \in R^{n \times n}$ is a symmetric and p.d. matrix, $Q(x) \in R^{n \times n}$ is a symmetric matrix, continuous with respect to $x \in R^{n}$, and $g(x) \in R^{n}$ is continuous with respect to $x$, then $\forall \rho \geq 0$ it is:

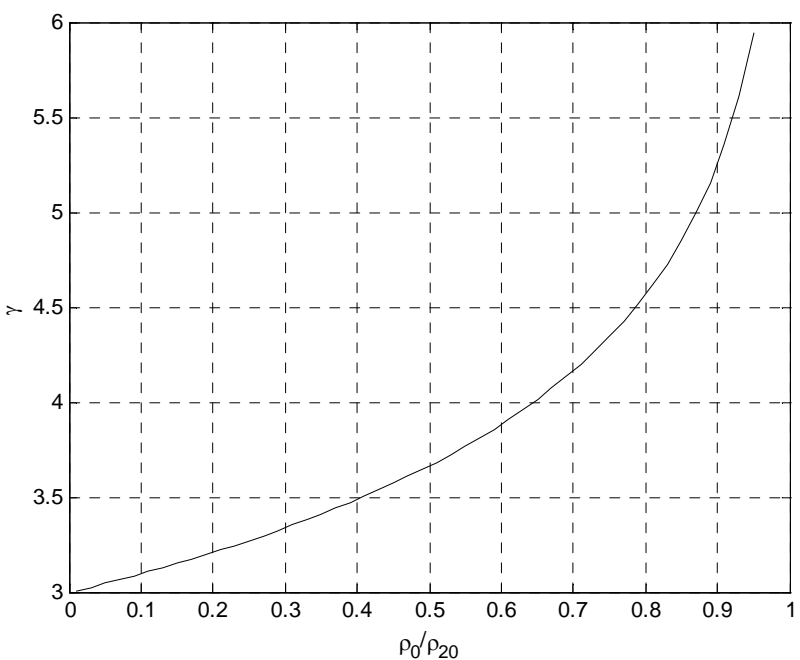

Figure 2. Factor $\gamma$ as a function of the normalized initial condition.

$$
\begin{aligned}
\min _{x \in C_{P, \rho}} x^{\mathrm{T}} Q(x) x & \geq \min _{x \in C_{P, \rho}} \lambda_{\min }\left(Q(x) P^{-1}\right) \rho^{2} \\
& \geq \min _{x \in \hat{C}_{P, \rho}} \lambda_{\min }\left(Q(x) P^{-1}\right) \rho^{2} \\
\max _{x \in C_{P, \rho}} x^{\mathrm{T}} g(x) & \leq \max _{x \in C_{P, \rho}} \sqrt{g(x)^{\mathrm{T}} P^{-1} g(x)} \rho \\
& \leq \max _{x \in \hat{C}_{P, \rho}} \sqrt{g(x)^{\mathrm{T}} P^{-1} g(x)} \rho .
\end{aligned}
$$

Moreover, if $Q(x)$ is linear with respect to $x$ it is

$$
\begin{aligned}
\min _{x \in C_{P, \rho}} x^{\mathrm{T}} Q(x) x & \geq \min _{x \in C_{P, 1}} \lambda_{\min }\left(Q(x) P^{-1}\right) \rho^{3} \\
& \geq \min _{x \in \hat{C}_{P, 1}} \lambda_{\min }\left(Q(x) P^{-1}\right) \rho^{3} .
\end{aligned}
$$

More in general, if $Q(x)$ is pseudo-linear with respect to $x$ with bounded coefficients, i.e. if $Q(x)=\sum_{i=1}^{n} Q_{i}(x) x_{i}$, where $Q_{i}(x)$ are bounded, then

$$
\begin{aligned}
& \min _{x \in C_{P, \rho}} x^{\mathrm{T}}\left(\sum_{i=1}^{n} Q_{i}(x) x_{i}\right) x \\
& \geq \min _{x \in C_{P, 1}, z \in R^{n}} \lambda_{\min }\left(\sum_{i=1}^{n} Q_{i}(z) x_{i} P^{-1}\right) \rho^{3} \\
& \geq \min _{x \in \hat{C}_{P, 1}, z \in R^{n}} \lambda_{\min }\left(\sum_{i=1}^{n} Q_{i}(z) x_{i} P^{-1}\right) \rho^{3} .
\end{aligned}
$$

Proof. Note that, if $f(x) \in R$ is a continuous function with respect to $x \in R^{n}$ and $X_{1} \subset X_{2}$ are compact subsets of $R^{n}$, it is $\min _{x \in X_{1}} f(x) \geq \min _{x \in X_{2}} f(x)$,

$\max _{x \in X_{1}} f(x) \leq \max _{x \in X_{2}} f(x)$. Moreover, since $P$ is $p . d$., there exists a symmetric nonsingular matrix $S$ such that $P=S^{2}$. Hence, by posing $z=S y$, it is 


$$
\begin{aligned}
& \min _{x \in C_{P, \rho}} x^{\mathrm{T}} Q(x) x \\
& \geq \min _{y \in C_{P, \rho}, x \in C_{P, \rho}} y^{\mathrm{T}} Q(x) y \\
& =\min _{z \in C_{I, \rho}, x \in C_{P, \rho}} z^{\mathrm{T}} S^{-1} Q(x) S^{-1} z \\
& =\min _{z \in C_{I, \rho}, x \in C_{P, \rho}} \lambda_{\min }\left(S^{-1} Q(x) S^{-1}\right) z^{\mathrm{T}} Z \\
& =\min _{x \in C_{P, \rho}} \lambda_{\min }\left(S S^{-1} Q(x) S^{-1} S^{-1}\right) \rho^{2} \\
& =\min _{x \in C_{P, \rho}} \lambda_{\min }\left(Q(x) P^{-1}\right) \rho^{2} \\
& \geq \min _{x \in \hat{C}_{P, \rho}} \lambda_{\min }\left(Q(x) P^{-1}\right) \rho^{2},
\end{aligned}
$$

and so (1.12) holds. Similarly

$$
\begin{aligned}
& \max _{x \in C_{P, \rho}} x^{\mathrm{T}} g(x) \\
& \leq \max _{y \in C_{P, \rho}, x \in C_{P, \rho}} y^{\mathrm{T}} g(x) \\
& =\max _{z \in C_{I, \rho}, x \in C_{P, \rho}} z^{\mathrm{T}} S^{-1} g(x) \\
& \leq \max _{z \in C_{I, \rho}, x \in C_{P, \rho}}\|z\|\left\|S^{-1} g(x)\right\| \\
& =\max _{x \in C_{P, \rho}} \sqrt{g(x)^{\mathrm{T}} P^{-1} g(x) \rho} \\
& \leq \max _{x \in \hat{C}_{P, \rho}} \sqrt{g(x)^{\mathrm{T}} P^{-1} g(x)} \rho,
\end{aligned}
$$

and hence (1.13). The inequalities (1.14) easily follow from the fact that, if $Q(x)$ is linear with respect to $x$, $\left.Q(x)\right|_{x \in C_{P, \rho}}=\left.Q(x)\right|_{x \in C_{P, 1}} \rho$. The inequalities (1.15) analogously follow.

Remark 1. Clearly, if $Q(x)$ and $g(x)$ are independent of $x,(1.12)$ and (1.13) are valid with the equal sign. If $Q(x)$ depends on $x, \min _{x \in C_{P, \rho}} x^{\mathrm{T}} Q(x) x$ is quite difficult to compute because $\left.x^{\mathrm{T}} Q(x) x\right|_{x \in C_{P, \rho}}$ has in general different points of relative maximum, of relative minimum and of "inflection"; moreover, the second and the third member of (1.12) (of (1.14) or of (1.15)) allow an easier computation of a lower bound on $\left.x^{\mathrm{T}} Q(x) x\right|_{x \in C_{P, \rho}}$ proportional to $\rho^{2}\left(\rho^{3}\right)$, as it will be shown later on. A similar talking is valid if $g(x)$ depends on $x$.

Lemma 2. Consider a p.d. matrix $P \in R^{n \times n}$ and a matrix $C \in R^{m \times n}$ with rank $m$. If $\|x\|_{P} \leq \rho$ then the smallest $\alpha$ such that $\|v\| \leq \alpha\|x\|_{P}$, where $v=C x$, is equal to $\alpha=\sqrt{\lambda_{\max }\left(C P^{-1} C^{\mathrm{T}}\right)}$.

Proof. Since $P$ is p.d., by posing $z=S x$, where $S$ is a symmetric nonsingular matrix such that $P=S^{2}$, then, in an equivalent way, the smallest $\alpha$ such that $\|v\| \leq \alpha\|x\|_{P} \leq \alpha \rho$ is also the smallest $\alpha$ such that the matrix $\alpha^{2} I-S^{-1} C^{\mathrm{T}} C S^{-1}$ is positive semidefinite, i.e. all its eigenvalues are non-negative. As

$$
\lambda\left(\alpha^{2} I-S^{-1} C^{\mathrm{T}} C S^{-1}\right)=\alpha^{2}-\lambda\left(S^{-1} C^{\mathrm{T}} C S^{-1}\right),
$$

it is $\alpha=\sqrt{\lambda_{\max }\left(S^{-1} C^{\mathrm{T}} C S^{-1}\right)}$. By taking into account that, if $F$ is a real $m \times n$ matrix with rank $m$, the matrix $F^{\mathrm{T}} F$ has $n-m$ null eigenvalues and $m$ positive eigenvalues equal to the ones of $F F^{\mathrm{T}}$, it follows that $\alpha=\sqrt{\lambda_{\max }\left(C S^{-1} S^{-1} C^{\mathrm{T}}\right)}=\sqrt{\lambda_{\max }\left(C P^{-1} C^{\mathrm{T}}\right)}$ and, hence, the proof.

Lemma 3. Let $P \in R^{n \times n}$ be a symmetric $p . d$. matrix with its inverse $P_{i}=P^{-1}$ having unitary elements on the main diagonal. Then the hyper-rectangle of $R^{n}$ externally tangent to the hyper-ellipse $E=\left\{x \in R^{n}: x^{\mathrm{T}} P x=1\right\}$ is the hyper-quadrilateral (quadrangle if $n=2$, cube if $n=3$ ) having as origin the centre and with unitary half-side.

Proof. It is easy to prove that the point of contact of the hyper-line orthogonal to the versor $e_{i}, i=1,2, \cdots, n$, of $R^{n}$ and tangent to the hyper-ellipse $E$ is

$$
p_{i}=\frac{P_{i} e_{i}}{\sqrt{e_{i}^{\mathrm{T}} P_{i} e_{i}}}=i \text {-th column of } P_{i} .
$$

From this the proof easily follows.

The following significant and useful theorem is stated.

Theorem 3. Let

$$
A=\sum_{i_{1}, i_{2}, \cdots, i_{l} \in\{0,1\}} A_{i_{1} i_{2}, \cdots, i_{l}} \pi_{1}^{i_{1}} \pi_{2}^{i_{2}}, \cdots, \pi_{l}^{i_{l}} \in R^{n \times n}
$$

be a matrix multilinearly depending on the parameters

$$
\left[\begin{array}{llll}
\pi_{1} & \pi_{2} & \cdots & \pi_{l}
\end{array}\right]^{\mathrm{T}}=\pi \in \Pi=\left\{\pi \in R^{l}: \pi^{-} \leq \pi \leq \pi^{+}\right\}
$$

and let $P \in R^{n \times n}$ be a symmetric p.d. matrix. Then the minimum (maximum) of $\lambda_{\pi \in \Pi}\left(Q P^{-1}\right)\left(\lambda_{\max }\left(Q P^{-1}\right)\right)$, where $Q=-\left(A^{\mathrm{T}} P+P A\right)$, is attained in one of the $2^{l}$ vertices of $\Pi$.

Proof. Note that for a constant $\pi_{j}, j \neq i$, it is $Q=Q_{0}+\pi_{i} Q_{1}, \pi_{i} \in\left[\pi_{i}^{-}, \pi_{i}^{-}\right]$. Moreover, by taking into account that $\lambda_{\min }\left(Q P^{-1}\right)=\min _{x \in\left\{x: x^{\mathrm{T}} P x=1\right\}} x^{\mathrm{T}} Q x$, it turns out to be that

$$
\begin{aligned}
& \min _{\pi_{i} \in\left[\pi_{i}^{-}, \pi_{i}^{+}\right]} \lambda_{\min }\left(\left(Q_{0}+\pi_{i} Q_{1}\right) P^{-1}\right) \\
= & \min _{\pi_{i} \in\left[\pi_{i}^{-}, \pi_{i}^{+}\right], x \in\left\{x: x^{\mathrm{T}} P x=1\right\}} x^{\mathrm{T}}\left(Q_{0}+\pi_{i} Q_{1}\right) x .
\end{aligned}
$$

Therefore, said $\hat{\pi}_{i}, \hat{x}$ the points of minimum of $f\left(\pi_{i}, x\right)=\left.x^{\mathrm{T}}\left(Q_{0}+\pi_{i} Q_{1}\right) x\right|_{x \in\left\{x: x^{\mathrm{T}} P x=1\right\}}$, it is 


$$
\begin{aligned}
& \min _{\substack{\pi_{i} \in\left[\pi_{i}^{-}, \pi_{i}^{+}\right]\\
}} \lambda_{\min }\left(\left(Q_{0}+\pi_{i} Q_{1}\right) P^{-1}\right) \\
= & \min _{\pi_{i} \in\left[\pi_{i}^{-}, \pi_{i}^{+}\right]}\left(\hat{x}^{\mathrm{T}} Q_{0} \hat{x}+\pi_{i} \hat{x}^{\mathrm{T}} Q_{0} \hat{x}\right) \\
= & \min _{\pi_{i} \in\left[\pi_{i}^{-}, \pi_{i}^{+}\right]}\left(\hat{c}_{0}+\pi_{i} \hat{c}_{1}\right)=\min \left\{\hat{c}_{0}+\pi_{i}^{-} \hat{c}_{1}, \hat{c}_{0}+\pi_{i}^{+} \hat{c}_{1}\right\} .
\end{aligned}
$$

From (1.20) the proof easily follows.

\section{Main Result}

Now the first main result, concerning the analysis of stability, can be stated.

Theorem 4. Give a symmetric p.d. matrix $P \in R^{n \times n}$. Then a majorant system of the system (1.4) is

$$
\dot{\rho}=\alpha_{1} \rho+\alpha_{2} \rho^{2}+\beta \delta, v=c \rho,
$$

in which:

$$
\begin{aligned}
& \alpha_{1}=-\min _{p \in V_{p}, g \in V_{g}} \frac{\lambda_{\text {min }}\left(Q_{1} P^{-1}\right)}{2}, \\
& Q_{1}=-\left(A_{1}^{\mathrm{T}} P+P A_{1}\right) \\
& \alpha_{2}=-\min _{x \in V_{P_{1}}, p \in V_{p}, g \in V_{g}} \frac{\lambda_{\min }\left(\sum_{i=1}^{n} Q_{2 i} x_{i} P^{-1}\right)}{2}, \\
& Q_{2 i}=-\left(A_{2 i}^{\mathrm{T}} P+P A_{2 i}\right) \\
& \beta=\sqrt{\lambda_{\max }\left(B^{\mathrm{T}} P B\right)}, \\
& c=\sqrt{\lambda_{\max }\left(C P^{-1} C^{\mathrm{T}}\right)} \\
& \delta \geq\|d\|,
\end{aligned}
$$

where $V_{p}$ and $V_{g}$ are the sets of vertices of the hyperrectangle $\wp$ and of the hyper-rectangle $\Upsilon$ respectively, and $V_{P_{1}}$ is the set of vertices of the hyper-rectangle of $R^{n}$ externally tangent to the hyper-ellipse $\left\{x \in R^{n}: x^{\mathrm{T}} P x=1\right\}$.

Proof. By choosing as "Lyapunov function" the quadratic form $V=x^{\mathrm{T}} P x=\|x\|_{P}^{2}=\rho^{2}$, for $x$ belonging to a generic hyper-circumference $C_{P, \rho}=\left\{x: x^{\mathrm{T}} P x=\rho^{2}\right\}$, it is

$$
2 \rho \dot{\rho} \leq \max _{x \in C_{P, \rho}, p \in \wp, t \in R}\left(-x^{\mathrm{T}} Q_{1} x-x^{\mathrm{T}}\left(\sum_{i=1}^{n} Q_{2 i} x_{i}\right) x+2 x^{\mathrm{T}} P B d\right) .
$$

The proof easily follows from (1.23), Lemmas 1, 2 and Theorem 3.

The second main result, concerning the synthesis of the stabilizing control law, follows from Theorem 4 .

\section{Theorem 5.}

Let $p_{a}(\lambda)=\beta_{v} a^{v}+\beta_{v-1} a^{v-1} \lambda+\cdots+\beta_{1} a \lambda^{v-1}+\lambda^{v} \quad$ be the characteristic polynomial of the low-pass Butterworth filter of order $v$ with cutoff frequency $\omega_{n}=a$.
If in (1.3) it is posed

$$
\begin{aligned}
& {\left[\begin{array}{llll}
K_{v} & K_{v-1} & \cdots & K_{1}
\end{array}\right]} \\
& =\left[\begin{array}{llll}
\beta_{v} a^{v} I & \beta_{v-1} a^{v-1} I & \cdots & \beta_{1} a I
\end{array}\right],
\end{aligned}
$$

in which $I$ is the identity matrix of order $m$, then a majorant system of the system (1.4) with respect to the norm $\|x\|_{P}$, with $P=T_{a} P_{1} T_{a}$, where

$$
\begin{aligned}
& P_{1}=\left(V V^{*}\right)^{-1}, \\
& V=\frac{1}{\sqrt{v}}\left[\begin{array}{cccc}
I & I & \cdots & I \\
\lambda_{1} I & \lambda_{2} I & \cdots & \lambda_{v} I \\
\vdots & \vdots & \ddots & \vdots \\
\lambda_{1}^{v-1} I & \lambda_{2}^{v-1} I & \cdots & \lambda_{v}^{v-1} I
\end{array}\right]=\frac{1}{\sqrt{v}}\left\{\lambda_{j}^{i-1} I\right\}, \\
& T_{a}=\left[\begin{array}{cccc}
a^{v-1} I & 0 & \cdots & 0 \\
0 & a^{v-2} I & \cdots & 0 \\
\vdots & \vdots & \ddots & \vdots \\
0 & 0 & \cdots & I
\end{array}\right]=\operatorname{diag}\left\{a^{n-i} I\right\}
\end{aligned}
$$

being $\lambda_{k}=\mathrm{e}^{i \frac{\pi}{2 v}(v+2 k-1)}, \quad k=1,2, \cdots, v$, the $k$-th root of $p_{a}(\lambda)$ for $a=1$, is

$$
\dot{\rho}=-a \gamma_{1 a} \rho+\gamma_{2 a} \rho^{2}+\beta \delta, v=\frac{1}{a^{v-1}} \rho,
$$

in which:

$$
\begin{aligned}
& \gamma_{1 a}=\min _{p \in V_{p}, g \in V_{g}} \frac{\lambda_{\min }\left(Q_{1 a} P_{1}^{-1}\right)}{2}, Q_{1 a}=-\left(A_{1 a}^{\mathrm{T}} P_{1}+P_{1} A_{1 a}\right), \\
& A_{1 a}=\left[\begin{array}{ccccc}
0 & I & 0 & \cdots & 0 \\
0 & 0 & I & \cdots & 0 \\
\vdots & \vdots & \vdots & \ddots & \vdots \\
0 & 0 & 0 & \cdots & I \\
\frac{F_{v}}{a^{v}}-\beta_{v} I & \frac{F_{v-1}}{a^{v-1}}-\beta_{v-1} I & \frac{F_{v-2}}{a^{v-2}}-\beta_{v-2} I & \cdots & \frac{F_{1}}{a}-\beta_{1} I
\end{array}\right] \\
& \begin{array}{l}
\gamma_{2 a}=-\min _{p \in V_{p}, g \in V_{g}, z_{i}= \pm 1} \frac{\lambda_{\min }\left(\sum_{i=1}^{n} Q_{2 a i} z_{i} P_{1}^{-1}\right)}{2}, \\
Q_{2 a i}=-\left(A_{2 a i}^{\mathrm{T}} P_{1}+P_{1} A_{2 a i}\right),
\end{array} \\
& A_{2 a i}=\frac{1}{a^{\left(v-1-\text { floor }\left(\frac{i-1}{m}\right)\right)}}\left[\begin{array}{cccc}
0 & 0 & \cdots & 0 \\
\vdots & \vdots & \ddots & \vdots \\
0 & 0 & \cdots & 0 \\
\frac{F_{2, i 1}}{a^{v-1}} & \frac{F_{2, i 2}}{a^{v-2}} & \cdots & \frac{F_{2, i v}}{1}
\end{array}\right], \\
& \beta=\sqrt{P_{1}(n, n)}=1.414,2.236,3.696,6.236, \cdots, \\
& n=2,3,4,5, \cdots \text {. }
\end{aligned}
$$


Proof. By making the change of variable $z=T_{a} x$, it is easy to prove that the system (1.4) becomes

$$
\begin{aligned}
& \dot{z}=a A_{1 a} z+\sum_{i=1, \cdots, n}\left(A_{2 a i} z_{i}\right) z+B d, \\
& y=C_{a} z=\left[I / a^{v-1} 0 \cdots 0\right] z .
\end{aligned}
$$

Moreover, note that the Butterworth eigenvalues $\lambda_{k}$ have unitary magnitudes; hence all the main diagonal elements of the matrix $V V^{*}=P_{1}^{-1}$ are unitary. From this consideration, from (1.30), from Lemmas 2, 3 and Theorem 4 , the proof easily follows.

Theorem 6. For $a \rightarrow \infty$, the parameters $\gamma_{1 a}$ and $\gamma_{2 a}$ of the majorant system (1.26) turn out to be

$$
\begin{aligned}
& \lim _{a \rightarrow \infty} \gamma_{1 a}=\hat{\gamma}_{1}=\cos \frac{v-1}{2 v} \pi=-\max _{i}\left(\operatorname{Real}\left(\lambda_{i}\right)\right) \\
& =0.7071,0.5000,0.3827,0.3090, \cdots \text {, } \\
& v=2,3,4,5, \cdots \\
& \lim _{a \rightarrow \infty} \gamma_{2 a}=\hat{\gamma}_{2}=-\min _{p \in V_{p}, g \in V_{g}, z_{i}= \pm 1} \frac{\lambda_{\min }\left(\sum_{i=m(v-1)+1}^{v m=n} Q_{2 i} Z_{i} P_{1}^{-1}\right)}{2}, \\
& Q_{2 i}=-\left(A_{2 i}^{\mathrm{T}} P_{1}+P_{1} A_{2 i}\right), A_{2 i}=\left[\begin{array}{cccc}
0 & 0 & \cdots & 0 \\
\vdots & \vdots & \ddots & \vdots \\
0 & 0 & \cdots & 0 \\
0 & 0 & \cdots & F_{2, i v}
\end{array}\right] .
\end{aligned}
$$

Proof. It is easy to verify that for $a \rightarrow \infty$ the matrix $V$ is the limit of the eigenvectors matrix of the matrix $A_{1 a}$ (see (1.27)); hence it is that $A_{1 \infty}=V \Lambda V^{-1}$, $A_{1 \infty}^{\mathrm{T}}=A_{1 \infty}^{*}=V^{*-1} \Lambda^{*} V^{*}$, where $\Lambda=\operatorname{diag}\left\{\operatorname{diag}\left\{\lambda_{1}, \cdots, \lambda_{1}\right\}, \cdots, \operatorname{diag}\left\{\lambda_{v}, \cdots, \lambda_{v}\right\}\right\}$. Therefore

$$
\begin{aligned}
& \lambda_{\min }\left(Q_{1} P_{1}^{-1}\right) \\
& =\lambda_{\min }\left(-A_{1 \infty}^{\mathrm{T}}-P A_{1 \infty} P^{-1}\right) \\
& =\lambda_{\min }\left(-V^{*-1} \Lambda^{*} V^{*}-V^{*-1} V^{-1} A_{1 \infty} V V^{*}\right) \\
& =\lambda_{\min }\left(-V^{*-1} \Lambda^{*} V^{*}-V^{*-1} \Lambda V^{*}\right)=-\lambda_{\max }\left(\Lambda^{*}+\Lambda\right),
\end{aligned}
$$

hence (1.31).

Moreover it is easy to verify that

$$
\begin{aligned}
\lim _{a \rightarrow \infty} A_{2 a i}= & 0, i=1,2, \cdots, m(v-1) ; \\
\lim _{a \rightarrow \infty} A_{2 a i}= & {\left[\begin{array}{cccc}
0 & \cdots & 0 & 0 \\
\vdots & \ddots & \vdots & \vdots \\
0 & \cdots & 0 & 0 \\
0 & \cdots & 0 & F_{2, i v}
\end{array}\right], i=m(v-1)+1, \cdots, m v . }
\end{aligned}
$$

From (1.28) and (1.34) the expression (1.32) easily follows.
Remark 2. If $m=1$ it is easy to prove that

$$
\begin{aligned}
& \lim _{a \rightarrow \infty} \gamma_{2 a}=\hat{\gamma}_{2}=\gamma_{2} \max \left|F_{2, v v}\right|, \\
& \gamma_{2}=\lambda_{\max }\left(\left(P_{1} e_{n} e_{n}^{\mathrm{T}}+e_{n} e_{n}^{\mathrm{T}} P_{1}\right) P_{1}^{-1} / 2\right) \\
& =1.207,1.618,2.348,3.618, \cdots, v=2,3,4,5, \cdots .
\end{aligned}
$$

From Theorems 5 and 6 the following result derives.

Theorem 7. Consider the system (1.4) with

$K_{1}, K_{2}, \cdots, K_{v}$ provided from (1.24). If the design parameter $a$ is big enough, from a practical point of view, the time constant $\tau_{l}$ of the linearized of the majorant system (1.26) is inverse proportional to $a$ and it coincides with the maximum time constant of the linearized of the system (1.4). More in detail, if $a$ is large enough it turns out to be

$$
\begin{aligned}
& \tau_{l}=\frac{1}{a \gamma_{1 a}} \cong-\frac{1}{\max \left(\operatorname{real}\left(\operatorname{eig}\left(A_{1 a}\right)\right)\right)} \cong \frac{\hat{\tau}_{l 1}}{a}, \\
& \hat{\tau}_{l 1}=1.414,2.000,2.613,3.236, \cdots, v=2,3,4,5, \cdots ;
\end{aligned}
$$

moreover, if $a$ is sufficiently large, for $t$ large enough it is

$$
\begin{aligned}
& \|y(t)\| \leq \frac{g}{a^{v}} \delta, \\
& g \cong \frac{\beta}{\hat{\gamma}_{1}}=2.000,4.472,9.657,20.180, \cdots, v=2,3,4,5, \cdots,
\end{aligned}
$$

or, more in general, it is

$$
\left\|y^{(i)}(t)\right\| \leq \frac{g}{a^{v-i}} \delta, i=0,1, \cdots, v-1 .
$$

Proof. (1.36) easily follows from (1.26), (1.31) and by noting that

$$
\lim _{a \rightarrow \infty} A_{1 a}=\left[\begin{array}{ccccc}
0 & I & 0 & \cdots & 0 \\
0 & 0 & I & \cdots & 0 \\
\vdots & \vdots & \vdots & \ddots & \vdots \\
0 & 0 & 0 & \cdots & I \\
-\beta_{v} I & -\beta_{v-1} I & -\beta_{v-2} I & \cdots & -\beta_{1} I
\end{array}\right] .
$$

The inequality (1.37) follows from (1.7), from the fact that if $a$ is sufficiently large then $\rho_{1} \cong \frac{\beta}{a \gamma_{1 a}} \delta$, from the second of (1.26) and from (1.29) and (1.31).

(1.38) analogously follows by taking into account that

$$
y^{(1)}=\dot{y}=\left[\begin{array}{llll}
0 & I & \cdots & 0
\end{array}\right] x, \cdots, y^{(v)}=\left[\begin{array}{llll}
0 & 0 & \cdots & I
\end{array}\right] x .
$$

\section{Examples}

The following examples show the utility and the efficiency of the results stated in the previous sections.

Example 1. Consider the pseudo-linear uncertain 
system

$$
\dddot{y}=-p_{1} \ddot{y}-\left(1+p_{1} \sin y\right) \dot{y}-p_{2} y+u+d,
$$

where $p_{1}=p_{2}=1 \% \pm 20 \%$ and $\left|d\left(t, y, \dot{y}, \ddot{y}, p_{1}, p_{2}\right)\right| \leq \delta$. By posing

$g_{1}=\sin y, \hat{p}_{1}=\hat{p}_{2}=1, \hat{g}_{1}=0, \hat{d}=0$ and by applying Theorem 5, the majorant system of the system (1.41) controlled with the control law

$u=\left(1-a^{3}\right) y+\left(1+\sin y-2 a^{2}\right) \dot{y}+(1-2 a) \ddot{y}$ turns out to be

$$
\dot{\rho}=-a \gamma_{1 a} \rho+2.236 \delta, v=\frac{1}{a^{2}} \rho .
$$

In Figure 3 the value of $\gamma_{1 a}$ for $a \in[1,20]$ is reported. It is significant to note that for $a \geq 6$ it is $\gamma_{1 a} \geq 0.9517$, i.e. $\gamma_{1 a}=0.5$ unless $5 \%$, in accord with Theorem 7. For $a=10$ it is $\gamma_{1 a}=0.4867$; hence $\tau_{l}=2.054 / 10=0.2054 \mathrm{~s}$. Moreover, being $\gamma_{2 a}=0$, it is $\rho_{1}=4.849 / a$ and $\rho_{2}=\infty$. Therefore, at "steady state", $\forall d:|d| \leq \delta$, it is:

$$
\begin{gathered}
|y| \leq 4.849 \delta / 10^{3} \cong 4.472 \delta / a^{3},|\dot{y}| \leq 4.849 \delta / a^{2} \text { and } \\
|\ddot{y}| \leq 4.849 \delta / a .
\end{gathered}
$$

Example 2. Consider the system of Figure 4 described by the equation

$$
\begin{aligned}
& \ddot{y}=\frac{1}{1000}\left[\begin{array}{cc}
-p_{1} & 0 \\
p_{1}-p_{3} & -p_{3}
\end{array}\right] \dot{y}+\frac{1}{1000}\left[\begin{array}{cc}
0 & p_{5} \\
0 & -2 p_{5}
\end{array}\right] y \\
& +\frac{1}{1000}\left[\begin{array}{cc}
-p_{2} s g\left(\dot{y}_{1}\right) & 0 \\
p_{2} s g\left(\dot{y}_{1}\right)-p_{4} s g\left(\dot{y}_{1}+\dot{y}_{2}\right) & -2 p_{4} s g\left(\dot{y}_{1}+\dot{y}_{2}\right)
\end{array}\right] \dot{y}_{1} \dot{y} \\
& +\frac{1}{1000}\left[\begin{array}{cc}
0 & 0 \\
0 & -p_{4} s g\left(\dot{y}_{1}+\dot{y}_{2}\right)
\end{array}\right] \dot{y}_{2} \dot{y} \\
& +\frac{1}{1000}\left[\begin{array}{rr}
1 & 0 \\
-1 & 1
\end{array}\right] u+\left[\begin{array}{rr}
1 & 0 \\
-1 & 1
\end{array}\right]\left[\begin{array}{l}
d_{1} \\
d_{2}
\end{array}\right]
\end{aligned}
$$

in which $d_{1}=-5+\delta_{1},\left|\delta_{1}\right| \leq 1, d_{2}=-5+\delta_{2},\left|\delta_{2}\right| \leq 1 \quad$ are the disturbance actions due to several causes included the slope of the road, $p_{1}=p_{3}=5 \% \pm 20 \%, p_{2}, p_{4} \in\left[\begin{array}{ll}0 & 2\end{array}\right]$, $p_{5}=1 \% \pm 20 \%$ and $\operatorname{sg}(\sigma)=2 / \pi a \tan (1000 \sigma) \cong \operatorname{sgn}(\sigma)$.

By posing $\hat{p}_{1}=\hat{p}_{3}=5, \hat{p}_{2}=\hat{p}_{4}=0, \hat{p}_{5}=1$, $\hat{d}_{1}=\hat{d}_{2}=-5$ and by applying Theorem 5 the majorant

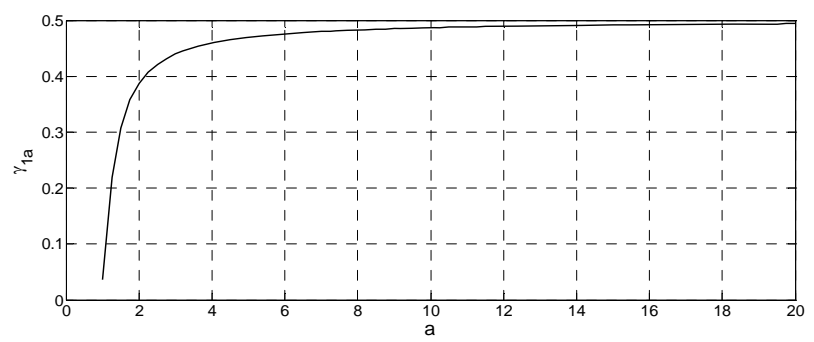

Figure 3. $\gamma_{1 a}$ as a function of $a$. system of (1.43), controlled by using the control law

$$
\begin{aligned}
u= & {\left[\begin{array}{ll}
1 & 0 \\
1 & 1
\end{array}\right]\left[\begin{array}{l}
-y_{2}+5 \dot{y}_{1} \\
2 y_{2}+5 \dot{y}_{2}
\end{array}\right]+\left[\begin{array}{rr}
1 & 0 \\
-1 & 1
\end{array}\right]\left[\begin{array}{l}
5 \\
5
\end{array}\right] } \\
& -1000\left[\begin{array}{ll}
1 & 0 \\
1 & 1
\end{array}\right]\left(a^{2} y+\sqrt{2} a \dot{y}\right),
\end{aligned}
$$

turns out to be

$$
\begin{aligned}
& \dot{\rho}=-a \gamma_{1 a} \rho+0.0079 \rho^{2}+1.414 \delta, \\
& \delta=\max \left\|\left[\begin{array}{rr}
1 & 0 \\
-1 & 1
\end{array}\right]\left[\begin{array}{l}
\delta_{1} \\
\delta_{2}
\end{array}\right]\right\|=2.236, v=\frac{1}{a} \rho .
\end{aligned}
$$

In Figure 5 the value of $\gamma_{1 a}$ for $a \in[1,20]$ is reported. It is significant to note that for $a \geq 13.5$ it turns out to be $\gamma_{1 a} \geq 0.6366$, i.e. $\gamma_{1 a}=0.7071$ unless $10 \%$, in accord with Theorem 7. For $a=10$ it is $\gamma_{1 a}=0.6111$; hence $\tau_{l}=0.1887 \mathrm{~s}$. Moreover it is $\rho_{1}=0.5177, \rho_{2}=$ 773.0. Therefore, at "steady state", $\forall \delta_{1}, \delta_{2}:\left|\delta_{1}\right| \leq 1$, $\left|\delta_{2}\right| \leq 1$, it is:

$$
\begin{gathered}
\|y\| \leq 0.05177 \cong 2 \times 2.236 / a^{2}, \\
\|\dot{y}\| \leq 0.5177 \cong 2 \times 2.236 / a .
\end{gathered}
$$

Figure 6 shows the values of $\|y(t)\|$ and of $\|\dot{y}(t)\|$, obtained for $p_{1}=5, p_{2}=1, p_{3}=5, p_{4}=1, p_{5}=1, \delta_{1}(t)$ and $\delta_{2}(t)$ square waves of amplitude 1 and frequency $1.2 \mathrm{~Hz}, x_{0}=\left[\begin{array}{llll}0.2 & 0.2 & 0 & 0\end{array}\right]^{\mathrm{T}}, \quad x_{0}=\left[\begin{array}{llll}0 & 0 & 0.2 & 0.2\end{array}\right]^{\mathrm{T}}$.

This figure highlights that the proposed stabilization method is little conservative, as it can be easily verified by simulating the stabilized system for several initial conditions and numerous values of the parameters.

\section{Conclusions}

In this paper the problem of analysis and practical

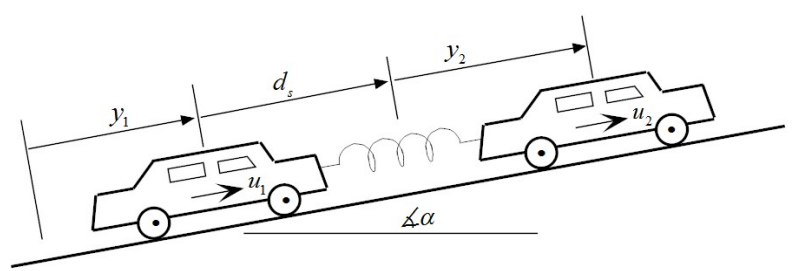

Figure 4. Vehicles to be kept still at a distance $d_{s}$.

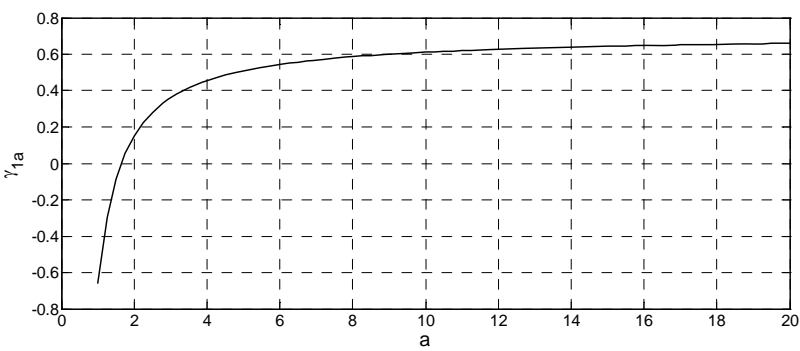

Figure 5. $\gamma_{1 a}$ as a function of $a$. 


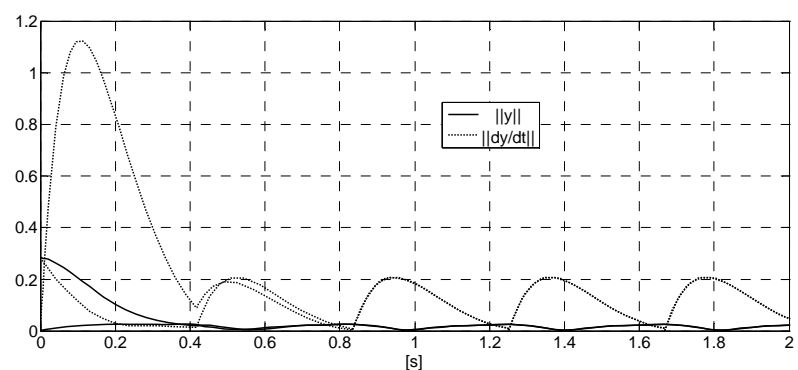

Figure 6. Possible time histories of $\|y(t)\|$ and of $\|\dot{y}(t)\|$.

stabilization of a significant class of MIMO nonlinear systems subject to parametric uncertainties, including linear and quadratic ones with an additional bounded nonlinearities and/or disturbances, has been approached. By using the concept of majorant system and via Lyapunov approach, new useful results, explicit formulas and efficient algorithms for designing state feedback control laws, with a possible imperfect compensation of nonlinearities and disturbances, have been stated. These results have been proved that guarantee a specified convergence velocity of the linearized of the majorant system and a desired steady-state output for generic uncertainties and/or nonlinearities and/or bounded disturbances.

The utility and the efficiency of the these results have been shown with two illustrative example.

The presented results can be used to establish further new useful theorems for the tracking of trajectories for relevant MIMO systems, like e.g. the robots.

In this direction the research of the author is going on.

\section{REFERENCES}

[1] G. Ambrosino, G. Celentano and F. Garofalo, "Tracking Control of High-Performance Robots via Stabilizing Controllers for Uncertain Systems," Journal of Optimization Theory and Applications, Vol. 50, No. 2, 1986, pp. 239255. doi:10.1007/BF00939271

[2] H. Nijmeijer and A. J. Van der Schaft, "Nonlinear Dynamical Control Systems," Springer-Verlag, Berlin, 1990. doi:10.1007/978-1-4757-2101-0

[3] J. J. E. Slotine and W. Li, "Applied Nonlinear Control," Prentice-Hall, Upper Saddle River, 1991.

[4] B. Brogliato and A. T. Neto, "Practical Stabilization of a Class of Nonlinear Systems with Partially Known Uncertainties," Automatica, Vol. 31, No. 1, 1995, 145-150. doi:10.1016/0005-1098(94)E0050-R

[5] R. A. Freeman and P. V. Kokotovic, "Robust Nonlinear Control Design,” Birkhauser, Boston, 1996. doi:10.1007/978-0-8176-4759-9

[6] A. Isidori, "Nonlinear Control Systems II," SpringerVerlag, New York, 1999.

doi:10.1007/978-1-4471-0549-7
[7] S. Sastry, "Nonlinear Systems, Analysis, Stability and Control," Springer-Verlag, New York, 1999.

[8] X.-Y. Lu and S. K. Spurgeon, "Output Feedback Stabilization of MIMO Non-Linear Systems via Dynamic Sliding Mode," International Journal of Robust and Nonlinear Control, Vol. 9, No. 5, 1999, pp. 275-305. doi:10.1002/(SICI)1099-1239(19990430)9:5<275::AID-R NC404>3.0.CO;2-F

[9] L. Moreau and D. Aeyels, "Practical Stability and Stabilization," IEEE Transactions on Automatic Control, Vol. 45, No. 8, 2000, pp. 1554-1558. doi:10.1109/9.871771

[10] I. Karafyllis and J. Tsinias, "Global Stabilization and Asymptotic Tracking for a Class of Nonlinear Systems by Means of Time-Varying Feedback," International Journal of Robust and Nonlinear Control, Vol. 13, No. 6, 2003, pp. 559-588. doi:10.1002/rnc.738

[11] L. Celentano, "A General and Efficient Robust Control Method for Uncertain Nonlinear Mechanical Systems," Proceedings of the IEEE Conference on Decision and Control, Seville, 12-15 December 2005, pp. 659-665. doi:10.1109/CDC.2005.1582231

[12] L. K. Murray and S. Jayasuriya, "An Improved NonSequential Multi-Input Multi-Output Quantitative Feedback Theory Design Methodology," International Journal of Robust and Nonlinear Control, Vol. 16, No. 8, 2006, pp. 379-395. doi:10.1002/rnc.1061

[13] E. Moulay and W. Perruquetti, "Finite Time Stability and Stabilization of a Class of Continuous Systems," Journal of Mathematical Analysis and Applications, Vol. 323, No. 2, 2006, pp. 1430-1443. doi:10.1016/j.jmaa.2005.11.046

[14] D. V. Efimov and A. L. Fradkov, "Input-to-Output Stabilization of Nonlinear Systems via Backstepping," International Journal of Robust and Nonlinear Control, Vol. 19, No. 6, 2009, pp. 613-633. doi:10.1002/rnc.1336

[15] M. L. Corradini, A. Cristofaro and G. Orlando, "Robust Stabilization of Multi-Input Plants with Saturating Actuators," IEEE Transactions on Automatic Control, Vol. 55, No. 2, 2010, pp. 419-425. doi:10.1109/TAC.2009.2036308

[16] S. F. Yang, "Efficient Algorithm for Computing QFT Bounds," International Journal of Control, Vol. 83, No. 4, 2010, pp. 716-723. doi:10.1080/00207170903390161

[17] F. Amato, C. Cosentino and A. Merola, "Sufficient Conditions for Finite-Time Stability and Stabilization of Nonlinear Quadratic Systems," IEEE Transactions on Automatic Control, Vol. 55, No. 2, 2010, pp. 430-434. doi:10.1109/TAC.2009.2036312

[18] S. Ding, C. Qian and S. Li, "Global Stabilization of a Class of Feedforward Systems with Lower-Order Nonlinearities," IEEE Transactions on Automatic Control, Vol. 55, No. 3, 2010, pp. 691-696. doi:10.1109/TAC.2009.2037455

[19] L. Celentano, "New Robust Tracking and Stabilization Methods for Significant Classes of Uncertain Linear and Nonlinear Systems," In: A. Mueller, Ed., Recent Advances in Robust Control-Novel Approaches and Design Methods, InTech, Winchester, 2011, pp. 247-270.

[20] L. Celentano, "Robust Tracking Controllers Design with 
Generic References for Continuous and Discrete Uncertain Linear SISO Systems," LAP_Lambert Academic Publishing, Saarbrücken, 2012.

[21] L. Celentano, "Robust Tracking Method for Uncertain
MIMO Systems of Realistic Trajectories," Journal of the Franklin Institute, Vol. 350, No. 3, 2013, pp. 437-451. doi:10.1016/i.jfranklin.2012.12.002 